\title{
ReaR

\section{Estudio APRICOT sobre la seguridad de los más pequeños}

Artículo original: Incidence of severe critical events in paediatric anaesthesia (APRICOT): a prospective multicentre observational study in 261 hospitals in Europe. Prof Walid Habre, MD, Nicola Disma, MD, Katalin Virag, MSc, Karin Becke, MD, Tom G Hansen, MD, Martin Jöhr, MD, et al. Published:March 28, 2017D0I:https://doi.org/10.1016/S2213-2600(17)30116-9 (PDF)

Navarro Echevarría P, Arnal Velasco D.

Hospital Universitario Fundación Alcorcón. Madrid. España.

\section{Resumen}

El manejo anestésico del paciente pediátrico requiere de una formación y unas estructuras específicas, debido a las situaciones adversas que se pueden producir. El estudio APRICOT (Anaesthesia PRactice In Children Obervational Trial) con una muestra de más de 30.000 pacientes de 33 países europeos y a través de un minucioso análisis observacional de cohortes prospectivo, recoge la naturaleza, la incidencia y los factores de riesgo de los evento críticos más comprometedores; desde los más comunes como el laringoespasmo o el broncoespasmo, la bradicardia hasta la parada cardiaca, pasando por fenómenos neurológicos, anafilaxia o errores de medicación. Obtiene unos resultados que distan en algunos aspectos de lo estudiado hasta ahora. La diversidad de los datos analizados permite identificar aquellos factores de riesgo relacionados con los eventos críticos, entre ellos el anestesista y el centro hospitalario. Pero sobre todo da pie a la necesidad de crear guías de manejo anestésico pediátrico encaminadas hacia una Anestesia más segura.

\section{Introducción}

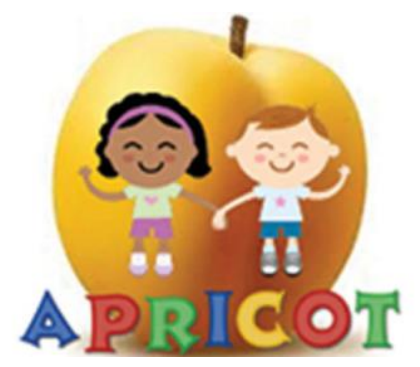

El manejo anestésico del paciente pediátrico requiere de una formación y unas estructuras específicas, debido a las situaciones adversas que se pueden producir. El estudio APRICOT (Anaesthesia PRactice In Children Obervational Trial) con una muestra de más de 30.000 pacientes de 33 países europeos y a través de un minucioso análisis observacional de cohortes prospectivo, recoge la naturaleza, la incidencia y los factores de riesgo de los evento críticos más comprometedores; desde los más comunes como el laringoespasmo o el broncoespasmo, la bradicardia hasta la parada cardiaca, pasando por fenómenos neurológicos, anafilaxia o errores de medicación. Obtiene unos resultados que distan en algunos aspectos de lo estudiado hasta ahora. La diversidad de los datos analizados permite identificar aquellos factores de riesgo relacionados con los eventos críticos, entre ellos el anestesista y el centro hospitalario. Pero sobre todo da pie a la necesidad de crear guías de manejo anestésico pediátrico encaminadas hacia una Anestesia más segura.

En los últimos años, se han desarrollado guías de manejo anestésico pediátrico y programas de formación específicos 
para la Anestesia Pediátrica con el fin de mejorar la seguridad en este ámbito. Sin embargo, poco sabemos sobre las situaciones más problemáticas que pueden surgir cuando nuestro paciente es un niño. Hace algo más de un año, salió a la luz un ambicioso estudio centrado en los eventos críticos graves del mundo de la Anestesia Pediátrica en Europa. Y es que, lo que hasta ahora sabíamos era gracias a estudios realizados en un sólo centro o en países específicos.

Posiblemente muchos anestesistas estábamos esperando un estudio como este para justificar nuestras decisiones cuando el parte del día está formado por niños: para muchos un reto, para otros su día a día. Puede que este artículo aclare muchas de nuestras dudas.

\section{Descripción del artículo}

Se trata de un estudio observacional de cohortes prospectivo multicéntrico cuyos objetivos son determinar la incidencia, naturaleza y resultados de los eventos críticos en los niños, así como la identificación de los factores de riesgo asociados.

Con la participación de 261 hospitales de 33 países europeos, el estudio contiene una muestra de 30.874 pacientes y 31.127 procedimientos anestésicos, recogida a lo largo de 2 semanas consecutivas en cada hospital entre abril de 2014 y enero de 2015 . Se analizaron los eventos críticos severos ocurridos en niños desde neonatos hasta los 15 años sometidos a procedimientos programados, urgentes o emergentes, bajo sedación, anestesia general, regional o combinada. Se excluyeron aquellos procedimientos realizados en unidades de Cuidados Críticos, y aquellos pacientes que acudieron intubados al quirófano. El seguimiento de los pacientes se llevó a cabo a la hora del episodio, al alta o a los 30 días. Los centros participantes fueron en su mayoría hospitales universitarios de adultos y niños, seguidos de hospitales puramente pediátricos, $y$ en menor medida hospitales comarcales y centros privados.

Los eventos críticos incluidos en el estudio fueron: laringoespasmo, broncoespasmo, aspiración pulmonar, errores de medicación, anafilaxia, inestabilidad cardiovascular, daño neurológico, parada cardiaca perioperatoria y estridor en el postoperatorio. Cada una de sus definiciones están disponibles en: http://www. esahq.org/research/clinicaltrialnetwork/ongoingtrials/apricot/document.

A través de un complejo análisis estadístico, se obtuvieron los siguientes resultados. La incidencia estimada de los eventos críticos severos en el estudio fue de $5,2 \%$ (IC $95 \%$ 5,05,5). Se emplearon diversos planes anestésicos: hasta 40 formas diferentes para premedicar, llegando a 100 las combinaciones de fármacos en la inducción.

Por otro lado, la mayor parte de los niños fueron ASA I y II. También se detalló la composición del equipo de Anestesia responsable siendo en la mayoría de los casos, independientemente de la clasificación ASA, un anestesista especializado en anestesia pediátrica (con experiencia en $>80 \%$ de casos pediátricos). Pero también pudieron ser atendidos por anestesistas con un $50-80 \%$ de casos pediátricos de experiencia, o bien por un anestesista con poca experiencia en casos con niños $(<50 \%)$ o incluso por un residente o enfermero o técnico de anestesia. Estos últimos atendieron $8,5 \%$ de pacientes ASA I; 7\% ASA II; $6,8 \%$ de casos ASA III y $2,6 \%$ ASA IV. (TABLA 1). 


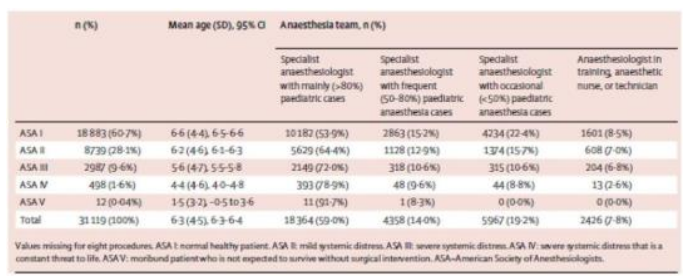

TABLA 1 (tabla tomada del artículo original). Media de edad de los pacientes en años (desviación estándar; intervalo de confianza al 95\%) y distribución según la clasificación ASA y el equipo anestésico.

En cuanto a la incidencia de los eventos críticos, los respiratorios ocurrieron con mayor frecuencia, seguidos de los cardiovasculares. La inducción y la educción constituyeron los dos momentos clave para la aparición de broncoespamo y laringoespamo, con una incidencia para ambos del 1,2\% (IC $95 \% 1,1-1,3)$. Por su parte, durante el mantenimiento se produjeron en mayor medida aquellos episodios de inestabilidad hemodinámica (hipotensión, sangrado, arritmias,...) cuya incidencia fue del 1,9\% (IC 95\% 1,7-2,0). (TABLAS 2 y 3 ). Los neonatos y lactantes (0-12 meses) presentaron con mayor frecuencia este tipo de eventos tanto respiratorios como cardiovasculares. En los niños mayores de 1 año tomó relevancia la aparición de eventos respiratorios con un descenso del riesgo del $12 \%$ por cada año de vida; mientras que los adolescentes fueron más vulnerables ante los eventos cardiovasculares. (FIGURA 1).

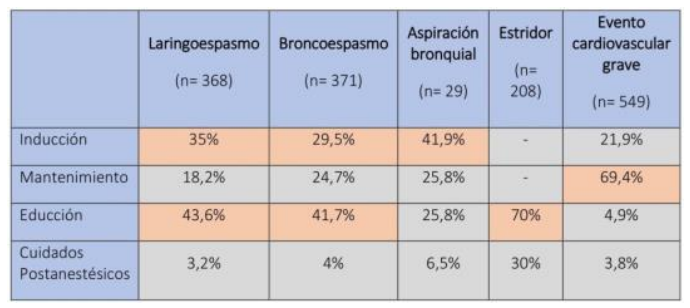

TABLA 2. Porcentaje de pacientes con eventos críticos respiratorios y cardiovasculares según el momento

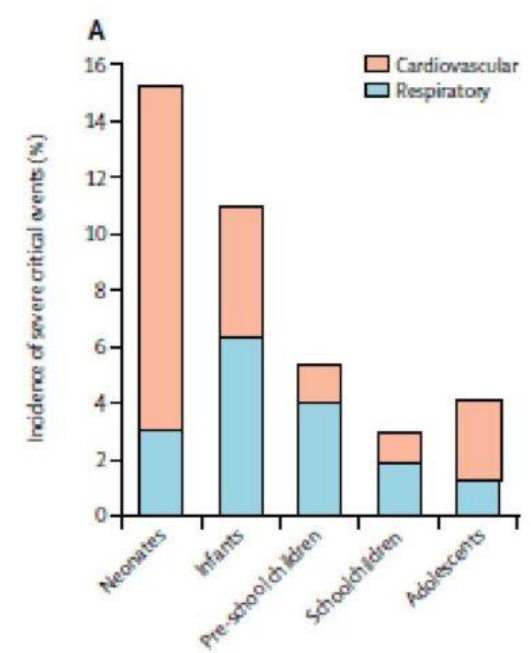

FIGURA 1 (tomada del artículo original). Distribución de los eventos críticos respiratorios y cardiovasculares por grupos de edad.

Respecto de los errores de medicación se estimó una incidencia del $0,2 \%$ (IC $95 \% 0,1-0,2)$, de ellos, un $59,2 \%$ lo constituyeron errores de dosificación con un desenlace en la mayoría de los casos sin secuelas o con mínimas secuelas. Fueron $10(0,03 \%$; IC $95 \%$ $0,01-0,05)$ las paradas cardíacas en 9 niños diferentes, causadas por hipoxemia y bajo gasto cardiaco principalmente. Cinco pacientes $(0,02 \%$; IC $95 \% 0,002-0,03)$ sufrieron daño neurológico y solamente hubo 3 casos de anafilaxia $(0,01 \%$; IC $95 \%$ $0,002-0,025)$. La cifra de mortalidad total descrita alcanza el $0,1 \%$ en los primeros 30 días, descartándose la anestesia como la causante. (TABLA $3)$. 


\begin{tabular}{|c|c|}
\hline Tipo de evento crítico & Incidencia \% (IC 95\%) \\
\hline Respiratorios: & \\
\hline$\bullet \quad$ laringoespasmo & $1,2 \%$ (IC 95\% 1,1-1,3) \\
\hline$\bullet \quad$ broncoespasmo & $1,2 \%$ (IC 95\% 1,1-1,3) \\
\hline bronquial & $0,1 \%$ (IC 95\% 0,06-0,13) \\
\hline bstridor & $0,7 \%$ (IC 95\% 0,6-0,8) \\
\hline Cardiovasculares & $1,9 \%$ (IC 95\% 1,7-2,0) \\
\hline Errores de medicación & $0,2 \%$ (IC 95\% 0,1-0,2) \\
\hline Daño neurológico & $0,02 \%$ (IC 95\% 0,002-0,03) \\
\hline Anafilaxia & $0,01 \%$ (IC 95\% 0,002-0,025). \\
\hline Parada cardiaca & $0,03 \%$ (IC 95\% 0,01-0,05) \\
\hline Mortalidad a los 30 días & $0,1 \%$ (IC 95\% 0,07-0,14) \\
\hline Incidencia total & $5,2 \%$ (IC 95\% 5,0-5,5). \\
\hline
\end{tabular}

TABLA 3. Incidencia (\%, IC 95\%) de los eventos críticos del estudio.

Los principales factores de riesgo identificados en el estudio fueron la edad, las comorbilidades (ASA), malformaciones o discapacidades, la hiperreactividad bronquial y el SAOS (Síndrome de Apnea Obstructiva del Sueño). El manejo anestésico fue también reconocido como factor de riesgo.

\section{Análisis crítico}

Los resultados obtenidos son cuanto menos interesantes, tanto por la cantidad de información que se puede exprimir de ellos como por el gran número de procedimientos que incluye el estudio. Resulta atractivo poder aplicar las cifras a los hospitales europeos en general, sin tener que extrapolarlos desde un centro concreto.

La mayoría de los resultados coinciden con trabajos previos, sobre todo en cuanto a los factores de riesgo respecta y la primacía de los eventos respiratorios frente a otros ${ }^{(1)}$. Pero llama particularmente la atención la incidencia de los eventos críticos severos estimada del $5,2 \%$ si la comparamos con otros estudios, a destacar el $0,14 \%$ que obtuvieron los americanos en $2014^{(2)}$. Posiblemente la razón sea que los estudios anteriores son retrospectivos ${ }^{(1)} \mathrm{o}$ basados en la comunicación voluntaria de incidentes ${ }^{(2)}$, mientras que en este caso se recogieron todos ellos, de manera anónima e imparcial para cada centro. Sin embargo, se podría identificar como limitación la diferente interpretación del evento adverso por cada anestesista implicado.

Las Recomendaciones para los Servicios de Anestesia Pediátrica de la Sociedad Europea de Anestesia Pediátrica y las de la Sociedad Española de Anestesia y Reanimación mencionan la evidente relación entre la disminución de la morbi-mortalidad en niños cuando el equipo responsable es experimentado ${ }^{(3)}$. Por lo que resulta sorprendente que haya $6,8 \%$ y $2,6 \%$ de pacientes ASA III y IV, respectivamente, que fueron atendidos por un residente o enfermero o técnico de anestesia. El estudio APRICOT va más allá y llega incluso a establecer los 3 años como la edad de corte por debajo de la cual el manejo anestésico debe ser llevado a cabo por personal experimentado, y más si el paciente posee alguno de los factores de riesgo identificados (malformaciones o discapacidades, la clasificación ASA, la hiperreactividad bronquial y SAOS). Es más, reconoce una mayor seguridad para estos procedimientos en centros con un amplio volumen de pacientes pediátricos, una mayor experiencia y unos medios adaptados.

La heterogeneidad y enorme diversidad existente en relación con el tipo de anestesia empleada en los diferentes procedimientos resulta positiva ya que permite identificar aquellas acciones que pueden resultar de mayor riesgo para el paciente. $\mathrm{Y}$ sobre todo, la tremenda variedad en el manejo de los niños genera la necesidad de unificar el manejo de los pacientes pediátricos. Esto implica el apoyo de las Sociedades 
Científicas pero necesita también del esfuerzo de cada centro.

\section{Conclusiones}

El estudio APRICOT aclara el reto que puede suponer la anestesia del paciente pediátrico, identifica factores que pueden ayudarnos a reducir los riesgos e incita a la implementación de guías de práctica clínica en Anestesia para el manejo de los más pequeños. En definitiva, anima a establecer estándares de cuidado y proyectos para mejorar la seguridad de los niños.

\section{Bibliografía}

1.Schleelein LE, Vincent AM, Jawad AF, Pruitt EY, Kreher GD, Rehman MA et al. Pediatric perioperative adverse events requiring rapid response: a retrospective case-control study.

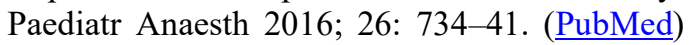
( $\underline{\text { HTML) }}$
2.Kurth CD, Tyler D, Heitmiller E, Tosone SR, Martin L, Deshpande JK. National pediatric anesthesia safety quality improvement program in the United States. Anesth Analg 2014; 119: 112-21. ( PubMed)

3.SEDAR. Sociedad Española de Anestesia, Reanimación y Terapéutica del Dolor. [Internet]. Recomendaciones para los Servicios de Anestesia Pediátrica de la SEDAR. Disponible en:

https://www.sedar.es/images/site/secciones/pedi atrica/recomendped.pdf

Correspondencia al autor

Patricia Navarro Echevarría pnavarroechevarria@gmail.com

MIR-4 Anestesia y Reanimación. Hospital Universitario Fundación Alcorcón.

Aceptado para el blog en abril de 2019 